\title{
Electric Field Dependence of Topological Edge States in One-Bilayer Bi(111): A First-Principles Study*
}

\author{
Hikaru Sawahata ${ }^{\dagger}$ and Naoya Yamaguchi \\ Graduate School of Natural Science and Technology, \\ Kanazawa University, Kanazawa 920-1192, Japan \\ Hiroki Kotaka \\ Elements Strategy Initiative for Catalysts and Batteries (ESICB), Kyoto University, Kyoto 615-8245, Japan \\ Fumiyuki Ishii ${ }^{\ddagger}$ \\ Nanomaterials Research Institute, Kanazawa University, Kanazawa 920-1192, Japan
}

(Received 9 January 2018; Accepted 29 October 2018; Published 24 November 2018)

\begin{abstract}
We investigate the effect of the electric field on the edge states in one-bilayer $\mathrm{Bi}(111)$ by first-principles calculations. We calculate the band structures of armchair and zigzag Bi nanoribbons. With increasing strength of the electric field $E>2.1 \mathrm{~V} / \AA$, the armchair nanoribbon shows a topological phase transition from non-trivial metallic edge states to insulating edge states. However, under the same conditions, the zigzag nanoribbon shows a topological phase transition from non-trivial metallic edge states to trivial metallic edge states. We expect that these findings will contribute to the development of, e.g., spin current switches for use in next-generation devices. [DOI: $10.1380 /$ ejssnt.2018.427]
\end{abstract}

Keywords: Bismuth; Topological insulator; Edge states; Density functional theory

\section{INTRODUCTION}

A topological insulator behaves as an insulating material in its bulk, but can conduct electrons along its surface. This behavior results from non-trivial metallic edge states due to its non-trivial topology being different from vacuum [1-3]. The origin of this non-trivial topology is band inversion induced by strong spin-orbit interactions; thus, many Bi alloys have been reported to be topological insulators [4-9].

One-bilayer $\mathrm{Bi}(111)$ is a typical two-dimensional topological insulating material. It has been theoretically predicted that $\mathrm{Bi}(111)$ is a topological insulator $[10,11]$ and films of this material have been experimentally formed on Si(111) substrates $[12,13]$. In addition, non-trivial edge states have been observed in $\mathrm{Bi}(111)$ formed on $\mathrm{Bi}_{2} \mathrm{Te}_{3}$ substrates [14]. Zigzag nanoribbons of one-bilayer $\mathrm{Bi}(111)$ show a pair of spin-degenerate bands originating from both edges. These bands are split by an electric field of $E=1.0 \mathrm{~V} / \AA[15]$. These non-trivial edge states are stable at an electric field $E<0.8 \mathrm{~V} / \AA$ [16].

Controlling spin currents on a topological insulator using an electric field expands the possibilities of using these materials for preparing novel devices [17]. In addition, as the non-trivial metallic edge states in topological insulators have dissipation-free spin currents, they could allow the development of spin current switches for use in nextgeneration devices [18]. In our previous study, we demonstrated that a topological phase of one-bilayer $\mathrm{Bi}(111)$ could be switched using an electric field of $2.1 \mathrm{~V} / \AA$, which was predicted by computing the topological invariant of the bulk system.

\footnotetext{
* This paper was presented at the 8th International Symposium on Surface Science, Tsukuba International Congress Center, Tsukuba, Japan, October 22-26, 2017.

$\dagger$ E-mail:sawahata@cphys.s.kanazawa-u.ac.jp

‡E-mail:ishii@cphys.s.kanazawa-u.ac.jp
}

In this study, we investigated the band structures of $\operatorname{Bi}(111)$ nanoribbons under an electric field by firstprinciples calculations and confirmed a topological phase transition from non-trivial edge states to trivial edge states. Using fully relativistic density functional calculations, we calculated the band structures in both armchair Bi nanoribbon (ABNR) and zigzag Bi nanoribbon (ZBNR) structures under an applied electric field. Analyzing the edge states in both nanoribbons upon application of a strong electric field, we observed that the nontrivial metallic edge states of ABNR changed to insulating states, whereas the non-trivial metallic edge states of ZBNR changed to trivial metallic edge states.

\section{METHOD}

Figure 1 shows the unit cell of one-bilayer Bi(111) structure where the direction of the applied electric field is indicated. We used an in-plane lattice constant of $4.54 \AA$ and buckling height of $1.45 \AA$, following a previous report of experimentally determined values [12]. Using OpenMX
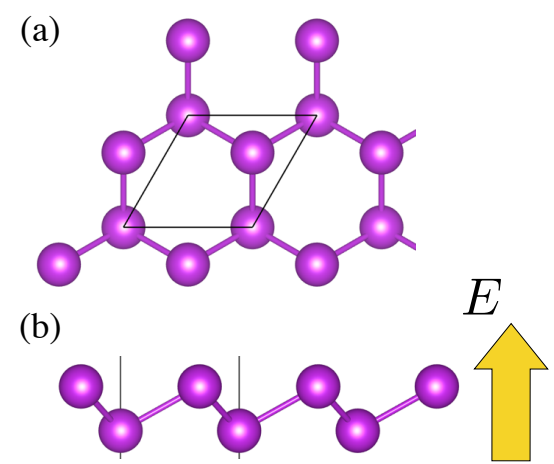

FIG. 1. Schematic diagram of the structure of one-bilayer Bi(111) film: (a) top view and (b) side view of the unit cell. The arrow shows the direction of the applied electric field. 


\section{(a) Armchair nanoribbon}

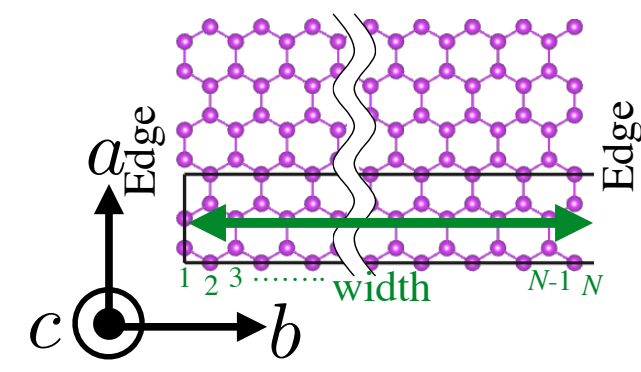

(b)

(c) Zigzag nanoribbon

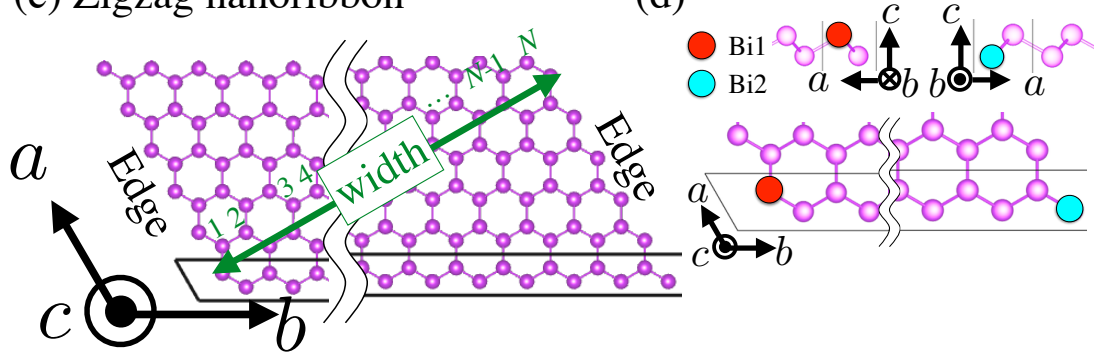

FIG. 2. The atomic structure and lattice vector of the (a) armchair nanoribbon and (c) zigzag nanoribbon used in this study. The edge atoms Bi1 and Bi2 are shown in (b) and (d), respectively. The width of a Bi nanoribbon is defined by $N$ in (a) and (c) for the armchair nanoribbon and the zigzag nanoribbon, respectively, where $N$ is the number of buckled Bi dimer lines.

(a)

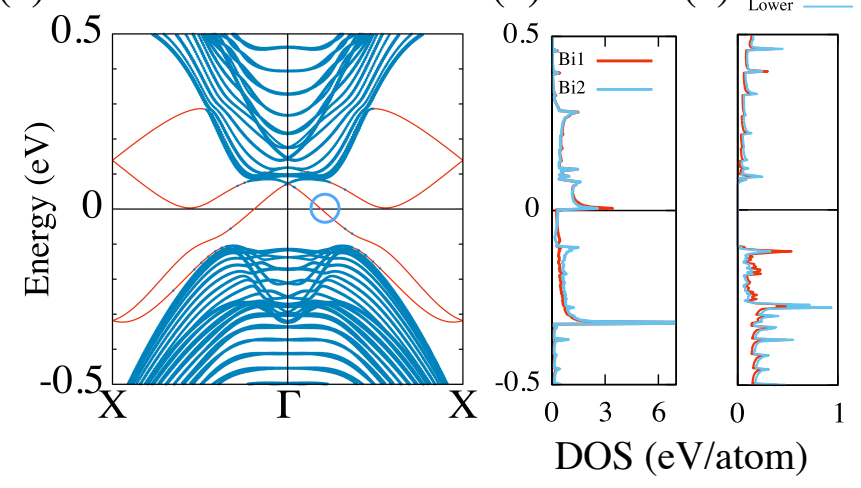

FIG. 3. (a) Band structures of the armchair nanoribbon at $E=1.0 \mathrm{~V} / \AA$. The blue lines indicate the band contribution from atoms inside the nanoribbons. The blue circles indicate the intersections of the Fermi level and band edges. (b) Partial density of states of edge atoms. Bi1 and Bi2 correspond to the edge atoms shown in Fig. 2(b). (c) Partial density of states of the upper and lower atoms inside the nanoribbons.

code [19], we performed fully relativistic density functional calculations for both ABNR and ZBNR structures. We used a local spin density approximation as an exchange correlation functional [20], norm-conserved pseudopotentials [21], and the linear combination of multiple pseudo-atomic orbitals for wave function expansion $[22,23]$. Spin-orbit interactions were considered using the fully relativistic total angular momentum $j$-dependent pseudopotentials [21]. The cutoff radius was 8.0 Bohr radius and pseudo-atomic orbitals were specified as s3p3d2 (i.e., three s-orbitals, three p-orbitals, and two d-orbitals). An energy cutoff of 300 Ry was used, with $\boldsymbol{k}$-space sampling points of $128 \times 1 \times 1$ for the reciprocal lattice vectors. The electric field was introduced as a saw-tooth potential (a)

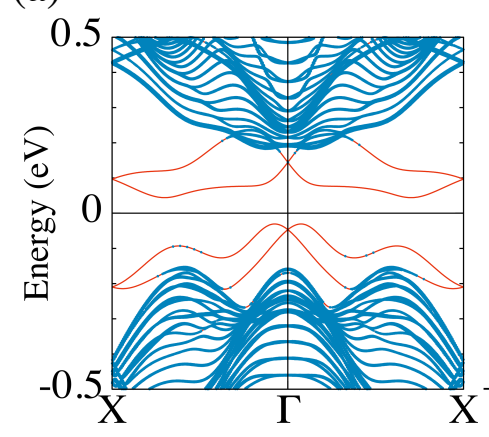

(b)

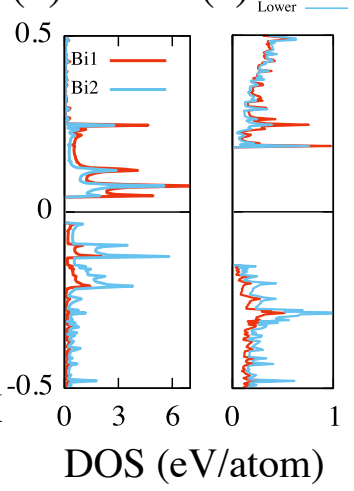

FIG. 4. (a) Band structures of the armchair nanoribbon at $E=4.0 \mathrm{~V} / \AA$. The blue lines indicate the band contribution from atoms inside the nanoribbons. (b) Partial density of states of edge atoms. Bi1 and Bi2 correspond to the edge atoms shown in Fig. 2(b). (c) Partial density of states of the upper and lower atoms inside the nanoribbons.

$[24,25]$. We did not consider changes in the lattice parameters and atomic positions induced by the electric field.

We considered $\mathrm{Bi}$ nanoribbon structures in the model in order to investigate the topological phase transition induced by an electric field. Figure $2(\mathrm{a}, \mathrm{b})$ shows the ABNR and ZBNR structures used in this study. The number of Bi atoms in the unit cell of ABNR and ZBNR was 128 and 64, respectively. The width of the ABNR and ZBNR was given by $N=64$ and 32 , respectively, where $N$ is the number of buckled $\mathrm{Bi}$ dimer lines, as shown in Figs. 2(a) and 2(c), respectively. The widths of ABNR and ZBNR in this study corresponded to $143.01 \AA$ and $145.28 \AA$, respectively [26]. Lattice vectors $a, b$, and $c$ were defined, where the vacuum space was $30 \AA$ and $20 \AA$ in the $b$ and $c$ directions, respectively, in both systems. 
To distinguish non-trivial edge states from trivial edge states, we counted the intersections between the Fermi level and edge bands. Topological insulators have gapless non-trivial edge states, which connect the conduction and valence bands, where each spin-resolved edge band crosses the Fermi level an odd number of times in the half Brillouin zone [27]. Hence, if the number of intersections divided by the number of edges (two) was odd, the system was non-trivial.

\section{RESULTS AND DISCUSSION}

Figures 3(a) and 4(a) show the electric-field dependence of the ABNR band structure, where the blue lines indicate the band contribution from atoms inside the nanoribbon. Figures 3(b) and 4(b) show the partial density of states (PDOS) projected on edge atoms [Bi1 and Bi2 shown in Fig. 2(b)], while Figs. 3(c) and 4(c) show the PDOS of the atoms inside the nanoribbon. We concluded that the bands around the Fermi level were mainly composed of edge states, as shown in the PDOS, as the PDOS of the $\mathrm{Bi}$ atoms inside the nanoribbons had a gap, in contrast to that of the edge atoms. The bandgap of the bulk was $0.2 \mathrm{eV}$ at an electric-field strength $E=1.0 \mathrm{~V} / \AA$ [Fig. 3(a)] and $0.35 \mathrm{eV}$ at $E=4.0 \mathrm{~V} / \AA$ [Fig. 4(a)]. This is consistent with the results of our previous study of a bulk onebilayer Bi system [18]. In the case of the band structure at $E=1.0 \mathrm{~V} / \AA$, the edge states were non-trivial as two degenerated edge bands intersected the Fermi level once between the $\Gamma$ and $\mathrm{X}$ points. On the other hand, in the band structure at $E=4.0 \mathrm{~V} / \AA$, the edge states had a bandgap of $0.02 \mathrm{eV}$ and showed a trivial insulating phase.

Figures $5(\mathrm{a}, \mathrm{b})$ and $6(\mathrm{a}, \mathrm{b})$ show the electric-field de-

(a)

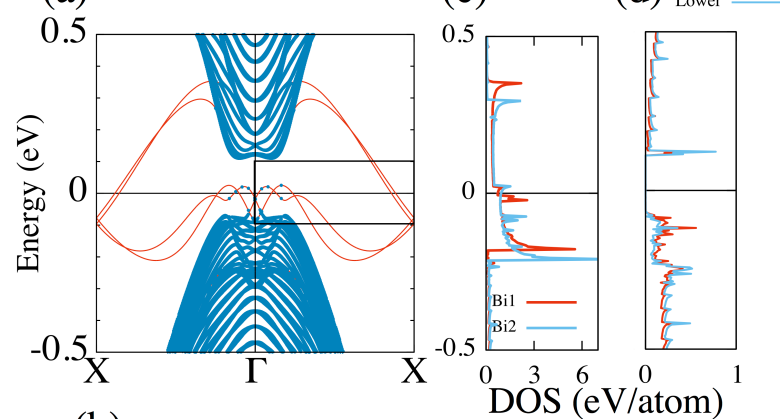

(b)

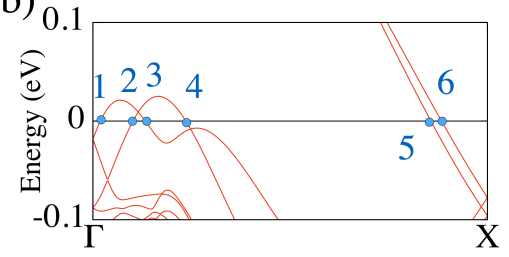

pendence of the band structure of ZBNR, where the blue lines indicate the bands contributed by atoms inside the nanoribbon. Figures 5(c) and 6(c) show the PDOS projected on edge atoms [Bi1 and Bi2 shown in Fig. 2(d)], while Figs. 5(d) and 6(d) show the PDOS of the atoms inside the nanoribbon. The bands around the Fermi level were mainly composed of edge states. The bandgap of the bulk was $0.2 \mathrm{eV}$ at $E=1.0 \mathrm{~V} / \AA$ [Fig. $5(\mathrm{a})$ ] and $0.39 \mathrm{eV}$ at $E=4.0 \mathrm{~V} / \AA$ [Fig. 6(a)]. These results were similar to those of ABNR and a previously measured bulk onebilayer system [18]. Considering the band structure at $E=1.0 \mathrm{~V} / \AA$ shown in Fig. 5(b), the edge states were non-trivial as the edge bands intersected the Fermi level six times between the $\Gamma$ and $\mathrm{X}$ points, i.e., the edge states for each edge intersected the Fermi level three times. On the other hand, as shown in Fig. 6(b), at $E=4.0 \mathrm{~V} / \AA$ trivial edge states were observed as two edge bands intersected the Fermi level eight times between the $\Gamma$ and $\mathrm{X}$ points, i.e., the edge states for each edge intersected the Fermi level four times.

We found that the non-trivial edge states of both ABNR and ZBNR could be switched to trivial edge states by applying an electric field of $4.0 \mathrm{~V} / \AA$; the ABNR switched from metallic to insulating behavior, while ZBNR remained metallic. A previous first-principles study revealed the existence of non-trivial edge states in $\mathrm{ABNR}$ and $\mathrm{ZBNR}$ without an electric field [11]. In the case of ZBNR, it was reported that the non-trivial edge states existed even under an applied electric field $E<1.0 \mathrm{~V} / \AA[15,16]$. Our results corresponded with those of our previous study of a bulk system where onebilayer $\operatorname{Bi}(111)$ showed a topological phase transition with an electric field $E>2.1 \mathrm{~V} / \AA$ [18]. It is interesting that the two nanoribbon structures showed different edge

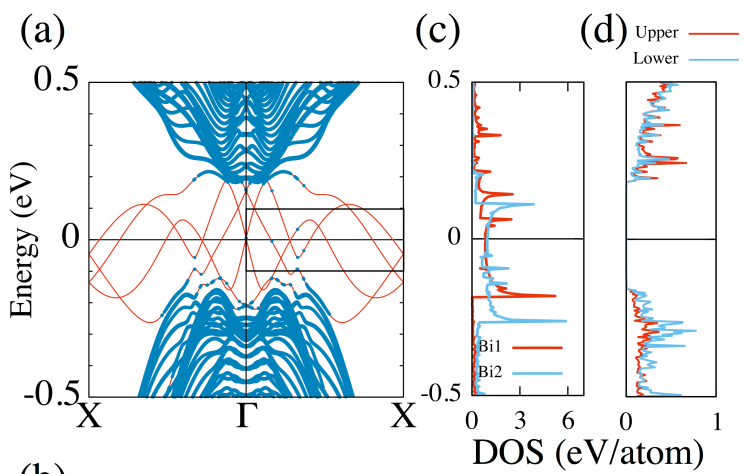

(b)

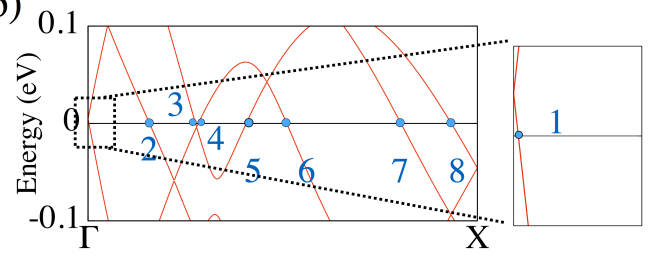

FIG. 5. (a) Band structures of the zigzag nanoribbon at $E=$ 1.0 $\mathrm{V} / \AA$. The blue lines indicate the band contribution from atoms inside the nanoribbons. (b) Enlarged view of the area indicated by the black box in (a). Six intersections between the Fermi level and band edges are shown. (c) Partial density of states of edge atoms. Bi1 and Bi2 correspond to the edge atoms shown in Fig. 2(d). (d) Partial density of states of the upper and lower atoms inside the nanoribbons.

FIG. 6. (a) Band structures of the zigzag nanoribbon at $E=4.0 \mathrm{~V} / \AA$. The blue lines indicate the band contribution from atoms inside the nanoribbons. (b) Enlarged view of the area indicated by the black box in (a). Eight intersections between the Fermi level and band edges are shown. (c) Partial density of states of edge atoms. Bi1 and Bi2 correspond to the edge atoms shown in Fig. 2(d). (d) Partial density of states of the upper and lower atoms inside the nanoribbons. 
states under a high electric field, resulting in only the ABNR showing the metal-insulator transition, while the ZBNR remained metallic. Such behavior is reminiscent of the electronic structure of graphene nanoribbons. In the case of hydrogenated graphene nanoribbons, the armchair nanoribbon has a bandgap, whereas the zigzag nanoribbon has flat metallic edge states [28, 29].

Generally, the topological phase transition is induced by band inversion. The origin of the topological insulating phase of one-bilayer $\mathrm{Bi}(111)$ is band inversion originating from strong spin-orbit interactions [10]. Under an applied electric field, further band inversion occurs and the system returns to the trivial insulating phase [18]. The origin of this band inversion is charge transfer between upper and lower Bi atoms in Fig. 1(b). In our calculations of the Bi nanoribbon, similar band inversion occurred in bulklike states. In Figs. 3(c) and 5(d), the PDOS of the upper atom was larger than that of the lower atom of the valence band near the Fermi level. In Figs. 4(c) and 6(d), the PDOS of the lower atom was larger than that of the upper atom of the valence band near the Fermi level.

\section{CONCLUSION}

We calculated the band structures of armchair and zigzag nanoribbons in one-bilayer $\mathrm{Bi}(111)$ and analyzed the edge states in order to investigate the topological phase transition induced by an electric field. The initial non-trivial edge states of both nanoribbons disappeared under an applied electric field. These results are consis- tent with those of one-bilayer $\mathrm{Bi}(111)$ showing a topological phase transition under an electric field of $2.1 \mathrm{~V} / \AA$ confirmed by computing a $Z_{2}$ topological invariant for the bulk [18]. When increasing the electric field strength up to $E=4.0 \mathrm{~V} / \AA$, the $\mathrm{ABNR}$ showed a transition from non-trivial metallic edge states to trivial insulating states, whereas ZBNR showed a transition from non-trivial to trivial metallic edge states. These findings are expected to contribute to a better understanding of the behavior of Bi nanostructures, which may assist in the development of novel devices using this material. These findings show that ZBNR structures are suitable for devices switching between charge current and spin current, while the ABNR structures are suitable for switching spin currents.

\section{ACKNOWLEDGMENTS}

This work was supported by Grant-in-Aid for Scientific Research on Innovative Area, "Nano Spin Conversion Science" (Grant No. 17H05180). This work was also supported by JSPS Grant-in-Aid for Scientific Research on Innovative Areas, "Discrete Geometric Analysis for Materials Design" (Grant No. 18H04481). The work was partially supported by Grants-in-Aid on Scientific Research under Grant No. 16K04875 from Japan Society for the Promotion of Science. The computations in this research were performed using the supercomputers at RIIT, Kyushu University, and the ISSP, University of Tokyo.
[1] C. L. Kane and E. J. Mele, Phys. Rev. Lett. 95, 146802 (2005).

[2] L. Fu and C. L. Kane, Phys. Rev. B 74, 195312 (2006).

[3] M. Z. Hasan and C. L. Kane, Rev. Mod. Phys. 82, 3045 (2010).

[4] J. C. Y. Teo, L. Fu, and C. L. Kane, Phys. Rev. B 78, 045426 (2008).

[5] D. Hsieh, D. Qian, L. Wray, Y. Xia, Y. S. Hor, R. J. Cava, and M. Z. Hasan, Nature 452, 970 (2008)

[6] H. Zhang, C.-X. Liu, X.-L. Qi, X. Dai, Z. Fang, and S.C. Zhang, Nat. Phys. 5, 438 (2009).

[7] Y. Xia, D. Qian, D. Hsieh, L. Wray, A. Pal, H. Lin, A. Bansil, D. Grauer, Y. S. Hor, R. J. Cava, and M. Z. Hasan, Nat. Phys. 5, 398 (2009).

[8] Y. L. Chen, J. G. Analytis, J.-H. Chu, Z. K. Liu, S.K. Mo, X. L. Qi, H. J. Zhang, D. H. Lu, X. Dai, Z. Fang, S. C. Zhang, I. R. Fisher, Z. Hussain, and Z.-X. Shen, Science 325, 178 (2009).

[9] D. Xiao, Y. Yao, W. Feng, J. Wen, W. Zhu, X.-Q. Chen, G. M. Stocks, and Z. Zhang, Phys. Rev. Lett. 105, 096404 (2010).

[10] S. Murakami, Phys. Rev. Lett. 97, 236805 (2006).

[11] M. Wada, S. Murakami, F. Freimuth, and G. Bihlmayer, Phys. Rev. B 83, 121310 (2011).

[12] T. Nagao, J. T. Sadowski, M. Saito, S. Yaginuma, Y. Fujikawa, T. Kogure, T. Ohno, Y. Hasegawa, S. Hasegawa, and T. Sakurai, Phys. Rev. Lett. 93, 105501 (2004).

[13] S. Yaginuma, K. Nagaoka, T. Nagao, G. Bihlmayer, Y. M. Koroteev, E. V. Chulkov, and T. Nakayama, J.
Phys. Soc. Jpn. 77, 014701 (2008).

[14] T. Hirahara, G. Bihlmayer, Y. Sakamoto, M. Yamada, H. Miyazaki, S.-i. Kimura, S. Blügel, and S. Hasegawa, Phys. Rev. Lett. 107, 166801 (2011).

[15] H. Kotaka, F. Ishii, M. Saito, T. Nagao, and S. Yaginuma, Jpn. J. Appl. Phys. 51, 025201 (2012).

[16] L. Chen, Z. F. Wang, and F. Liu, Phys. Rev. B 87, 235420 (2013).

[17] Q. Liu, X. Zhang, L. Abdalla, A. Fazzio, and A. Zunger, Nano Lett. 15, 1222 (2015).

[18] H. Sawahata, N. Yamaguchi, H. Kotaka, and F. Ishii, Jpn. J. Appl. Phys. 57, 030309 (2018).

[19] T. Ozaki et al., http://www.openmx-square.org/.

[20] J. P. Perdew and A. Zunger, Phys. Rev. B 23, 5048 (1981).

[21] G. Theurich and N. A. Hill, Phys. Rev. B 64, 073106 (2001).

[22] T. Ozaki, Phys. Rev. B 67, 155108 (2003).

[23] T. Ozaki and H. Kino, Phys. Rev. B 69, 195113 (2004).

[24] K. Kunc and R. Resta, Phys. Rev. Lett. 51, 686 (1983).

[25] R. Resta and K. Kunc, Phys. Rev. B 34, 7146 (1986).

[26] The width of ABNR (ZBNR) can be calculated by $(N-$ 1) $/ 2 \times 4.54 \AA(N \times 4.54 \AA)$.

[27] L. Fu and C. L. Kane, Phys. Rev. B 76, 045302 (2007).

[28] M. Fujita, K. Wakabayashi, K. Nakada, and K. Kusakabe, J. Phys. Soc. Jpn. 65, 1920 (1996).

[29] K. Nakada, M. Fujita, G. Dresselhaus, and M. S. Dresselhaus, Phys. Rev. B 54, 17954 (1996). 